PHYSICAL REVIEW A 91, 019902(E) (2015)

\title{
Publisher's Note: Temporal dissipative solitons in a three-level atomic medium confined in a photonic-band-gap fiber [Phys. Rev. A 91, 013828 (2015)]
}

M. Facão, S. Rodrigues, and M. I. Carvalho

(Received 22 January 2015; published 29 January 2015)

DOI: 10.1103/PhysRevA.91.019902

PACS number(s): 42.81.Dp, 42.50.Gy, 42.65.Hw, 99.10.Fg

This paper was published online on 20 January 2015 with errors on page 4. On page 4, in the right-hand column, the third line of the second inline equation should read as

$$
\frac{d T_{0}}{d Z}=-k, \quad \frac{d \zeta}{d Z}=\frac{1}{2}\left(\eta^{2}-k^{2}\right)+T_{0} \frac{d k}{d Z}
$$

The following equation on the same page should read as

$$
\eta_{e}=\sqrt{\frac{3\left(\xi^{2}+4 \alpha v\right)}{4 v(v-2 \sigma)}}, \quad k_{e}=-\frac{\xi}{2 v}
$$

The paper has been corrected as of 22 January 2015. The text is correct in the printed version of the journal. 